Review

\title{
The Biocompatibility of Nanodiamonds and Their Application in Drug De- livery Systems
}

\author{
Ying Zhu' ${ }^{1}$, Jing Li ${ }^{1}$, Wenxin $\mathrm{Li}^{1}$, Yu Zhang ${ }^{1}$, Xiafeng Yang ${ }^{1,2}$, Nan Chen ${ }^{1}$, Yanhong Sun ${ }^{1}$, Yun Zhao², Chunhai \\ Fan1, Qing Huang ${ }^{1 凶}$
}

1. Laboratory of Physical Biology, Shanghai Institute of Applied Physics, Chinese Academy of Sciences, Shanghai 201800, China;

2. School of Life Sciences, Sichuan University, Sichuan 610064, China.

$\bowtie$ Corresponding author: Telephone: +86-21-39194520; Fax: +86-21-59553021 E-mail: huangqing@sinap.ac.cn.

(C) Ivyspring International Publisher. This is an open-access article distributed under the terms of the Creative Commons License (http://creativecommons.org/ licenses/by-nc-nd/3.0/). Reproduction is permitted for personal, noncommercial use, provided that the article is in whole, unmodified, and properly cited.

Received: 2011.10.12; Accepted: 2011.12.22; Published: 2012.03.07

\begin{abstract}
Nanodiamonds (NDs), as a new member of the carbon nanoparticles family, have attracted more and more attention in biomedicine recently due to their excellent physical and chemical properties. This paper summarizes the main results from the in vitro and in vivo safety assessments of NDs and reports the application of NDs in the development of drug delivery systems. In view of the NDs' characteristics of easy formation of a porous cluster structure in solution, an adsorption model for a variety of functional molecules on the ND clusters is proposed, which provides new ideas for developing a novel smart drug with various features such as sustained-release, targeting, and fluorescence imaging.
\end{abstract}

Key words: Nanodiamonds (NDs), biocompatibility, drug delivery system, adsorption model, versatility.

\section{Introduction}

Nanodiamonds (NDs) are carbon nanoparticles with a truncated octahedral architecture that are typically about 2 to $8 \mathrm{~nm}$ in diameter. They not only exhibit various superior characteristics of diamond, such as chemical stability, and extremely high hardness, stiffness and strength, but also have the advantages of nanomaterials, such as small size, large surface area, and high adsorption capacity. Therefore, NDs have superior physical and chemical properties over conventional materials. Furthermore, detonation of carbon-containing explosives can be used in an inexpensive synthesis of NDs on a large scale. When an oxygen-deficient explosive mixture of trinitrotoluene (TNT)/hexogen (RDX) is detonated in a closed chamber, NDs are formed at the front of detonation wave in a time period of several microseconds. The yield of NDs via detonation is crucially dependent on the synthesis condition, especially on the heat capacity of the cooling medium (water, air, $\mathrm{CO}_{2}$, etc.) in the detonation chamber. The higher the cooling capacity, the larger the diamond's yield will be [1]. These advantageous properties of NDs have attract extensive research interests.

In fact, as early as in 1963, a high concentration of NDs was found for the first time in the smoke after the explosion of an explosive named Mixture B used by the army. Although it has been studied in the military secrecy for a long time, it was not until 1990s that NDs have started mass production and been widely used in basic researches [1]. At present, a large variety of applications for NDs have been proposed, including electrochemical coatings, polymer compositions, 
antifriction coatings, polishing, lubricants, biosensors, imaging probes, implant coatings and drug carriers [2-8]. Therefore, in view of their widespread applications and industrial production $[1,4,8]$, it is necessary to understand the bio-compatibility of NDs in either cell-based systems or animal models. This paper, in its first part, summarizes the main results from the in vitro and in vivo safety assessments of NDs, and further points out that NDs can be highly up-taken by cells and have good biocompatibility, which are thus widely used in the biomedical applications in recent years. In the second part of this paper, application of NDs in the development of drug delivery systems is reported. Finally, an adsorption model for a variety of functional molecules on NDs is proposed herein in view of the NDs' characteristics that it is easy to form a porous cluster structure spontaneously in solution [9]. This adsorption model provides an outlook of the strategies based on NDs for developing a novel smart drug delivery system with the functional features such as slow-release, targeting, and fluorescence imaging, and also contributes an important document basis for building drug delivery nanosystems with high efficiency and low toxicity.

\section{The Biocompatibility of Nanodiamonds In Vitro Cytotoxicity}

In the carbon nanomaterial safety assessment, in vitro cytotoxicity test is an important research subject because it is simple, reproducible, and easy to control the consistency of experimental conditions. Schrand et al. first reported cellular toxicity of NDs through MTT assay and ATP production assay and found that NDs do not induce significant toxicity on a variety of cells [10]. In their follow-up work, they compared the toxicity of NDs (with a single-particle size of 2-10 nm), carbon black (CB), multi-walled carbon nanotubes (MWNT), and single-walled carbon nanotubes (SWNT) on neuroblastoma cells and macrophages, and found that the toxicity follows the order of SWNT $>$ MWNT > CB > ND (see figure 1). In addition, the macrophage cells were more greatly affected by the presence of carbon nanomaterials, generating up to five times the amount of reactive oxygen species (ROS) compared with that generated from the neuroblastoma cells after exposure to either MWNT or SWNT. However, there was a lack of ROS generation from either cell line after incubation with the NDs as well as intact mitochondrial membranes, which further supported the notion that NDs were of low toxicity [11]. Similarly, Liu et al. measured the toxicity of NDs and carbon nanotubes (CNTs) on human lung A549 epithelial cells and HFL-1 normal fibroblasts, and their results indicated that at the concentration of 0.1-100 $\mu \mathrm{g} / \mathrm{ml}$, two kinds of carboxyl-modified NDs with a particle size of $5 \mathrm{~nm}$ and $100 \mathrm{~nm}$ respectively did not reduce the cell viability or alter the protein expression profile. In contrast, carboxyl-modified CNTs exposed under the same conditions showed a significant toxicity to the cells. They further compared the cytotoxicity of uncarboxylated NDs and CNTs on A549 cells, and obtained similar results [12]. Recently our group evaluated the biocompatibility of the detonation NDs. As consistent with the results reported in the literatures, we found that NDs in complete cell culture media showed no apparent toxicity on Hela cells, and pointed out that the cytotoxicity of NDs was highly related to serum proteins present in the cell culture medium [9].
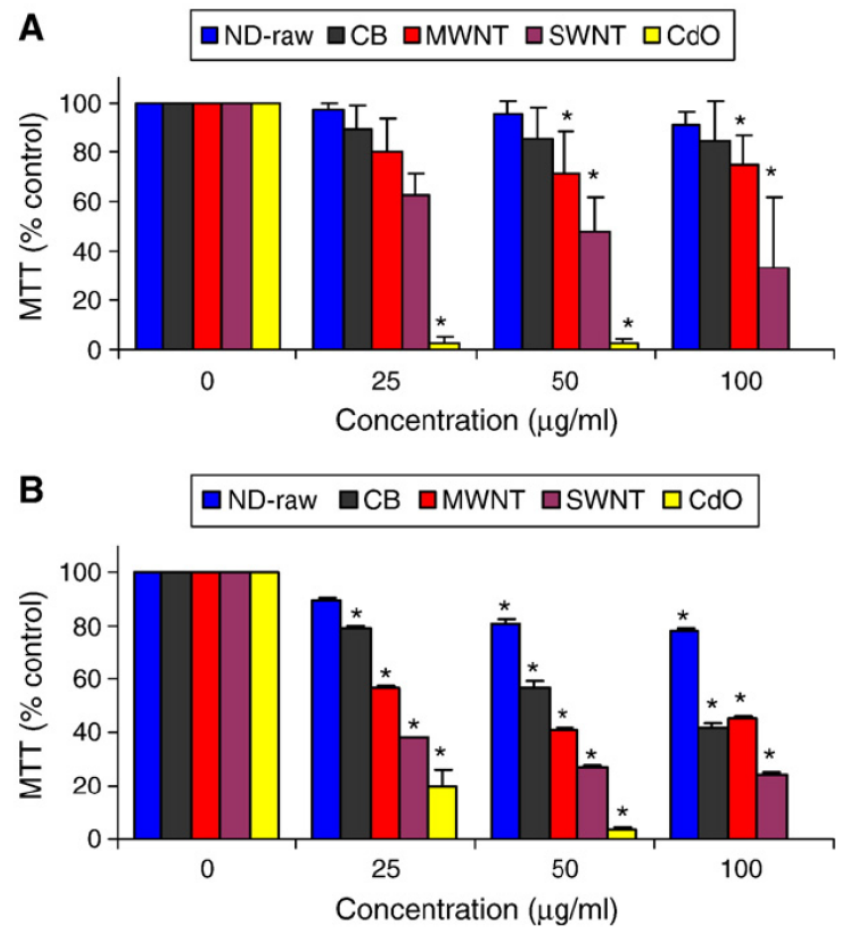

Figure I. Cytotoxicity evaluation after $24 \mathrm{~h}$ of incubation with various nanocarbons showing differential toxicities on (A) neuroblastoma cells or (B) macrophages. Values that are significantly different from the control $(p<0.05)$ are denoted with asterisks $(*)$. [Reprinted from ref. II with permission].

Genotoxicity means that the action of a variety of factors in the environment on an organism causes the injury of its genetic materials at a chromosome level, a molecular level or a base level, resulting in toxic effects. It is an important subject of the in vitro safety assessment of carbon nanoparticles. The chromosomal 
damage caused by interaction between some carbon nanoparticles and cells can be self-healing and result in no genotoxicity on the organism, whereas the chromosomal or genetic damage caused by other nanoparticles can be irreversible and result in genotoxicity. Huang et al. found no significant change in the expression of TNF $a$ and Bcl-x genes after incubation with the acid purified NDs compared with controls. Additional study of morphological changes and DNA fragmentation in macrophages as well as the viability of HT-29 human colorectal adenocarcinoma cells revealed a high biocompatibility of the NDs [7]. Furthermore, Liu et al. incubated A549 lung cancer cells and 3T3-L1 embryonic fibroblasts with NDs for a long time (10 days), and found that the cell viability was not altered. ND particles taken up by cells were almost equally separated into two daughter cells of cell division, and individual cells retained a single ND cluster in the cytoplasm after sub-cultured for several generations. The ND cluster within the cell didn't induce damages even after a long-term cell culture. Moreover, ND particles did not interfere with the gene or protein expressions on the regulation of cell cycle progression and adipogenic differentiation of a variety of cancer cell lines. Therefore, it was concluded that endocytic ND particles are non-cytotoxic during cell division and differentiation [13]. However, study on the genotoxicity of NDs by Xing et al. gave a different result, indicating that incubation of embryonic stem cells with NDs led to a slightly increased expression of DNA repair proteins (p53 and MOGG-1) and the oxidized NDs (O-NDs) which caused more DNA damage than the pristine/raw NDs (R-NDs). This suggests that the chemical groups introduced onto NDs by modification can lead to certain genotoxicity. However, compared with MWNT, the DNA damages caused by either the O-NDs or the R-NDs are much less severe [14]. In the CNT cytotoxicity assessment, Magrez et al. also pointed out that the toxicity of CNTs increased after acidic purification. In their opinion, grafting additional chemical groups such as carbonyl, carboxyl and hydroxyl groups, which are putatively "toxic", onto the surface of CNTs reduced the number of viable cells [15]. Due to the poor water-solubility of raw CNTs, reflux in a concentrated acid is generally required before experiments. However, the raw NDs typically have a good water-solubility even without any treatment such as acidic modification. Therefore, any oxidative modification of NDs needs to be minimized in the experiment, while keeping the NDs in the original form to afford a minimal side effect.

As a member of carbon nanomaterials, detonation NDs with very small particle sizes tend to form aggregates in solution which have a lower surface free energy and a particle size of tens to hundreds of nanometers. Therefore, they are easily taken up by cells. Zhang et al. indicated that in comparison with CNTs and graphene, NDs had the highest cellular uptake rate [16]. Generally speaking, ND particles were taken into cells via a clathrin-mediated endocytosis pathway [13]. Vaijayanthimala et al. investigated the uptake mechanism of NDs in the cancer cells (HeLa) and pre-adipocytes (3T3-L1) in detail. They also confirmed that the uptake of NDs in both cells was through an energy-dependent clathrin-mediated endocytosis. In addition, the surface charge of ND influences its cellular uptake, as the uptake of poly-L-lysine-coated NDs is better than that of oxidative-acid-purified NDs at the same concentration. A large number of NDs into the cells do not affect the in vitro differentiation of 3T3-L1 pre-adipocytes and 489-2 osteoprogenitors [17]. As can be seen from the in vitro studies, NDs can be highly up-taken by cells and show a good biocompatibility with a variety of cells.

\section{In Vivo Toxicity}

In comparison with the in vitro cellular experiment, the results from the in vivo biocompatibility evaluation using animal models can further reflect the potential impact of NDs on the environment and human health. NDs from the detonation synthesis are in the form of a powder with a low density, and therefore tend to spread into the air during the manufacturing and processing to cause environmental pollution. Therefore, the study of their toxicity on the respiratory system is particularly important. Intratracheal instillation is an important route to study the toxicity of nanoparticles on the respiratory system. For the first time, Wang group studied the pulmonary toxicity of the NDs administered by intratracheal instillation in mice, and confirmed that NDs did not have noticeable adverse effects in the lungs within the study period according to the histopathological and ultrastructural investigations. Furthermore, no lipid peroxidation of the lung was observed. Thus the intratracheally instilled NDs are of low pulmonary toxicity. Through the HRTEM images of NDs in the digested pulmonary tissues, they also examined the in vivo distribution and translocation of NDs after intratracheal instillation, and found NDs existed in the alveoli and bronchia at different time points. The results indicated that engulfment by lung macrophages might be the most important way to remove NDs [18]. Recently, we also studied the acute toxicity of NDs administrated by intratracheal instillation, and the histomorphology analysis and related biochemical indicators showed a dose-dependent toxicity of NDs 
in the lung, liver, kidney and blood [19]. The concentrations of NDs used in our experiment were $0.8,4$ and $20 \mathrm{mg} / \mathrm{kg}$, whereas those in Wang's experiment were 0.1 and $1 \mathrm{mg} / \mathrm{kg}$. The maximum concentration of NDs in our experiment was 20 times that in Wang's experiment. As can be seen, although NDs have a good biocompatibility, they are still a foreign non-degradable material for biological organisms and may bring the organisms side effects when their exposure concentration exceeds a certain range. However, the respiratory toxicity of NDs, even under the highest dose, on organisms is much smaller than that of CNTs and other carbon nanomaterials reported in the previous literature [20-22].

In addition to intratracheal instillation, Puzyr et al. used oral administration to study the long-term toxicity of ND hydrosols on mice and the offspring thereof. In their experiment, water in the mice's diet was replaced by 0.002 to 0.05 wt. \% ND hydrosols for three to six months, and the total amount of NDs delivered to each mouse was between $16 \mathrm{mg}$ to $450 \mathrm{mg}$ depending on the concentration of NDs in hydrosols. Their results from comparison with the control mice indicated that NDs neither caused death nor affected the growth or the internal organ (liver, lungs, heart, kidneys, and pancreas) weight dynamics. Furthermore, the substitution of water with ND hydrosols did not affect the mouse's reproductive ability as at least the first three generations of the mice consuming ND hydrosols since birth had produced healthy offspring [23]. In another study, Puzyr et al. further observed no inflammatory symptoms in the mice after subcutaneous exposure to NDs for three months [24]. Bakowicz et al. reported that rats exhibited no immune responses on the tenth day after intraperitoneal injection of NDs [25]. It can be seen that NDs administrated in various routes show good biocompatibility with organisms.

After introducing nanomaterials into the body by various routes of administration, we need to further understand the specific mechanism of their interaction with living systems. The absorption, distribution, metabolism and excretion (ADME) process of nanomaterials in the living body is an important basis for studying the interaction. Since ND particles are very small, it is difficult to observe their distribution in the animal organs by a conventional microscopy method. Radionuclide tracer technique can be applicable to the research in this field due to its easy detection, high sensitivity, good reliability, and almost no outside interference. At present, various radionuclides such as ${ }^{99 \mathrm{mTc}}[26,31],{ }^{67} \mathrm{Ga}[27],{ }^{125 \mathrm{I}}[28],{ }^{111} \mathrm{In}$
[29], and ${ }^{64} \mathrm{Cu}$ [30] have been used for labeling carbon nanoparticles. We used ${ }^{188}$ Re to label NDs. The labeled complex ${ }^{188} \mathrm{Re}-\mathrm{ND}$ has a radiochemical purity of more than $99 \%$ after several days, and is therefore suitable for experimental research. Biodistribution measurement showed that NDs administered by intratracheal instillation were distributed mainly in the spleen, liver, bones and heart in addition to the high retention in the lung [19]. However, no NDs were found by Wang et al. in the mice's liver, spleen and lymphatic node through histopathological and HRTEM observations, even when the mice were instilled with a much higher dose of $20 \mathrm{mg} / \mathrm{kg}$ NDs [18]. This difference may be due to different sources of NDs in these two studies. More importantly, the in vivo distribution data obtained by us using radionuclide labeling technique show that the distribution of NDs in the lung are much higher than that in other tissues or organs. Therefore, radionuclide labeling technique is much more sensitive, allowing detection of the information that cannot be obtained by electron microscopy. In another work of Wang's research group, the distribution of NDs after intravenous injection was studied in mice by using ${ }^{125}$ I-labeled NDs. The stability of ${ }^{125}$ I-NDs was greater than $90 \%$ within $25 \mathrm{~h}$, and the in vivo distribution result showed that NDs predominantly accumulated in the liver, spleen and lung. About $60 \%$ of the injected NDs were found in the mouse liver at $0.5 \mathrm{~h}$ post injection, and the level kept constant over 28 days [32]. Rojas et al. labeled NDs with another radionuclide ${ }^{18} \mathrm{~F}$ to study their in vivo biodistribution. By positron emission tomography, they intuitively observed the distribution of the NDs administrated via intravenous injection in various organs of the mice. Their results showed that the NDs were mainly distributed in lung, spleen and liver, and excreted into the urinary tract. Their research further indicated that addition of surfactant agents did not significantly change this distribution pattern, except for a slight reduction in the urinary excretion rate. It was also found that after removing those NDs with a larger particle size by filtration, the uptake of NDs was completely inhibited in the lung and spleen and significantly reduced in the liver (see figure 2) [33].

In summary, NDs have no or a small toxic side effect on biological systems. Under a different route of administration, the in vivo absorption, distribution, excretion and metabolism of NDs may be different, which allows us better understand the risk that NDs may pose to human health and provides an important basis for designing a ND-based drug delivery system. 

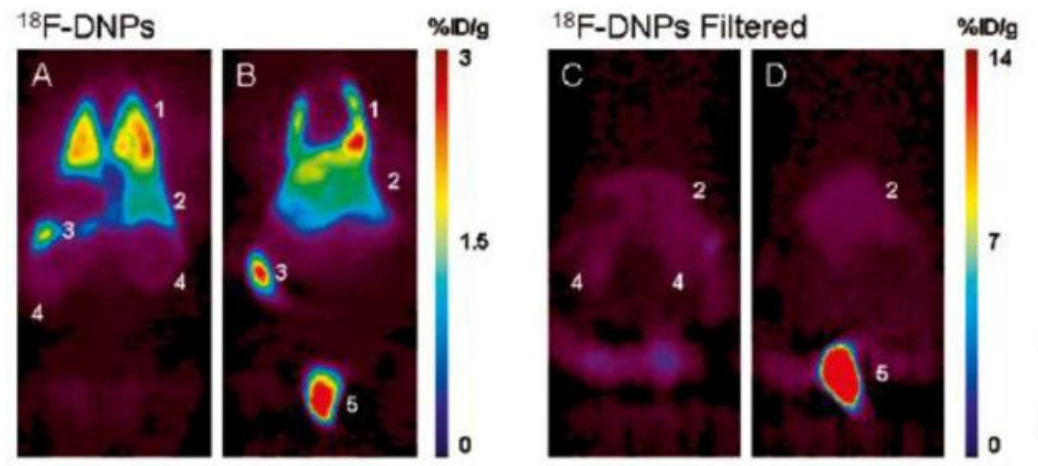

\section{1) Lung \\ 2) Liver}
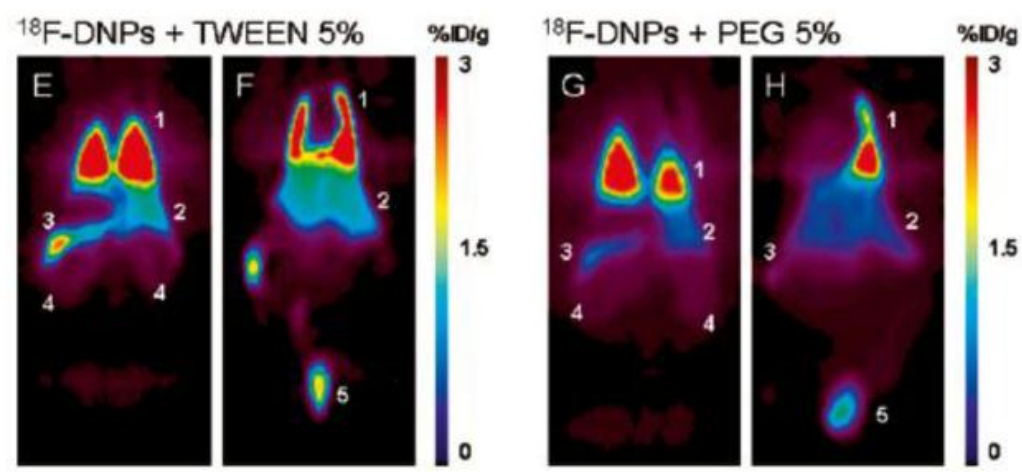

3) Spleen

4) Kidney

5) Bladder

Figure 2. Coronal sections of PET images acquired 120 min after injection of four different ND preparations. Images show animals injected with $18 \mathrm{~F}-\mathrm{NDs}$ dorsal $(\mathrm{A})$ to ventral $(\mathrm{B})$, with filtered $18 \mathrm{~F}-\mathrm{NDs}$ dorsal $(\mathrm{C})$ to ventral (D), with $18 \mathrm{~F}-\mathrm{NDs}+\mathrm{Tween} 80$ dorsal $(\mathrm{E})$ to ventral $(\mathrm{F})$, and finally with $18 \mathrm{~F}-\mathrm{NDs}+\mathrm{PEG}_{8000}$ dorsal $(\mathrm{G})$ to ventral $(\mathrm{H})$. Organs that exhibited an elevated uptake of the radiolabeled compound are labeled with numbers for easy identification. [Reprinted from ref. 33 with permission].

\section{Application in Drug Delivery Systems}

Since CNTs and other nanomaterials were discovered, they have been widely applied in the design of drug delivery systems because of their small sizes, easy penetration through cells, and their special structures on the surface which allow a covalent or non-covalent bond to the chemicals with poor membrane permeability such as small molecule drugs, peptides, protein drugs or DNA [34-38]. Current researches focus on the application of SWNT [35, 38], MWNT [34], and graphene [36,37] in drug delivery systems. However, many studies have reported that the CNTs and graphene exhibit some cytotoxicity, induce oxidative stress, and lead to apoptosis or necrosis [39-41]. NDs, as a new member of carbon nanoparticles family, have emerged as an alternative promising material for building drug delivery systems with high efficiency and low toxicity owing to their superior physico-chemical properties and good biocompatibility. Currently, NDs are used as a drug carrier mainly in two forms: (1) NDs assemble on a chemical substrate to form a thin film, having interactions with a drug in two-dimension; (2) NDs form spontaneous clusters also named as ND hydrogel with low free energy in an aqueous solution, having interactions with a drug in three-dimension.

\section{Applications of NDs Film in Drug Delivery Sys- tems}

The research work on ND-based film to build drug delivery systems has just started, and it is a new research field in the application of NDs in medicine. In many studies, ND films are prepared via a self-assembly process [42-45], wherein the preparation method reported by Dean Ho's research team is the simplest method [44]. They deposited ND hydrogels onto the poly-lysine (PLL) coated glass substrate through self-assembly and controlled the thickness of ND-PLL film via a layer-by-layer technique [44]. The investigation of cellular gene expression as well as MTT and DNA fragmentation assays revealed that the film has good biocompatibility. Various biological molecules can then assemble on the film (Figure 3). The experiment showed that such a self-assembly of dexamethasone, which was a kind of glucocorticoid, exhibited obvious anti-inflammatory effects on the RAW264.7 cells, and the dexamethasone loaded on the NDs film had a slow-release effect. Therefore, 
their toxic side effects on the normal tissues were effectively reduced [44]. In another study, NDs bound with DOX were embedded in a Parylene C polymer microfilm. The microfilm architecture consists of DOX-ND conjugates sandwiched between a base layer of Parylene $C$ and a thin variable layer of Parylene $C$, which allows for modulation of drug release. Due to the powerful sequestration ability of the DOX-ND complex and the release-modulating nature of the thin Parylene layer, the microfilms displayed stable and continuous slow-release of drugs for at least one month. Through DNA fragmentation assays, the authors demonstrated that this film retained the activity of DOX under biological conditions [46].

Only very few studies have been reported on building drug delivery systems based on ND films, which may be due to their limited applications at present. A ND-drug film can be implanted immediately after surgical removal of a tumor to target residual cancerous cells so as to effectively prevent the tumor from recurring. In addition, a ND-drug film can be more applicable to the treatment of superficial tumors such as breast cancer, head and neck cancer, and skin cancer, or superficial skin inflammations, wherein the drug is delivered transdermally to tumors or inflammation sites, reducing the toxicity on normal tissues.

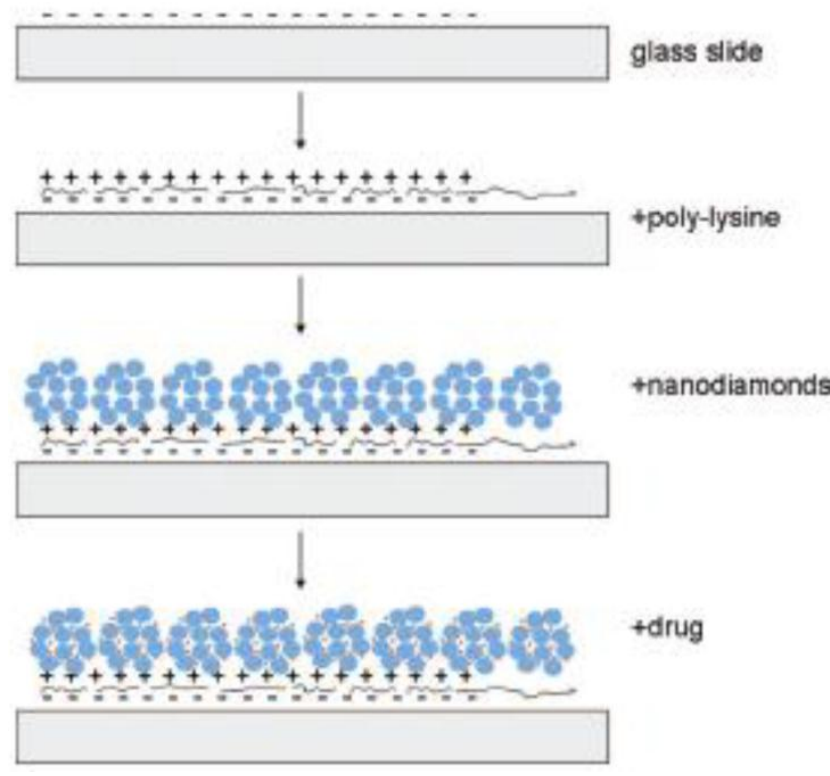

Figure 3. Schematic drawing of the ND-nanofilm formation and the drug incorporation into the film. [Reprinted from ref. 44 with permission].

\section{Applications of ND Clusters in Drug Delivery Systems}

NDs dispersed in aqueous solution can spontaneously form clusters with a lower free energy, also named as hydrogel. The clusters have a size of tens to hundreds of nanometers. Drug delivery systems based on such clusters have been reported, wherein the drug is loaded onto ND clusters mainly in a non-covalent manner. Again, Dean Ho's group at the Northwestern University has done a number of pioneering studies. They investigated the adsorption of a water-insoluble anti-cancer drug doxorubicin (DOX) hydrochloride $[7,47,48]$, a protein kinase inhibitor purvalanol A (a highly promising medicament for liver cancer), 4-hydroxytamoxifen (a drug for the treatment of breast cancer), anti-inflammatory drug dexamethasone [49] and the diabetes drug insulin onto the carrier of ND hydrogel, and also examined the therapeutic efficacy of the resulting complexes [50].

In the preparation of ND-DOX complex, Huang et al. found that introducing a small inorganic molecule $\mathrm{NaCl}(10 \mathrm{mg} / \mathrm{ml})$ to the reaction system increased the adsorption of DOX on NDs from $0.5 \mathrm{wt} \%$ to $10 \mathrm{wt} \%$, and the toxicities of the complexes on RAW264.7 mouse macrophage and HT-29 human colon cancer cells were less than that of DOX group. The authors speculate that the ND aggregates have a shielding effect on DOX so that slow release of the drug protects normal cells or tissues from side effects posed by the drug [7]. They further examined the efficacy of the NDX (ND-DOX complex) in mouse models of liver cancer and mammary cancer. Biodistribution analysis showed that NDs can be cleared out of the body. More importantly, they found that NDX overcame the drug efflux from tumor cells, allowing anticancer drugs to kill drug-resistant cells at a lower dosage, thus lowering the drug toxicity to normal organs and tissues. Gradual release of the drug from NDX allowed for increased drug retention in blood circulation and tumor, therefore NDX significantly increased inhibition of apoptosis and tumor growth beyond the conventional DOX treatment in both tumor models (Figure 4) [47]. This research work provides a promising foundation for continued ND-based drug development and potential clinical applications [51]. Recently, the same research group studied the interaction process between ND clusters and DOX, and found that the binding of DOX molecules onto ND occurs only at a high $\mathrm{pH}$ and requires at least about $10 \%$ of the ND surface area to be fully titrated for the binding to occur [48]. 
A

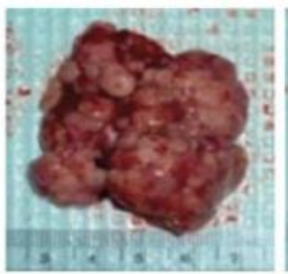

PBS

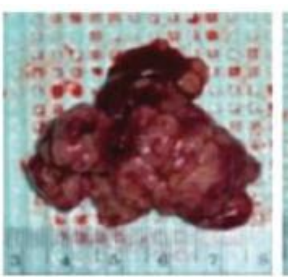

ND $400 \mu g$

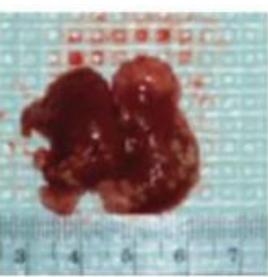

Dox $100 \mu \mathrm{g}$

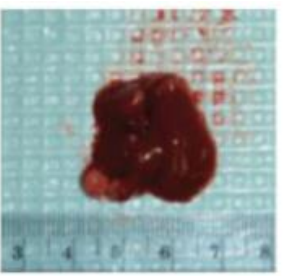

NDX $100 \mu \mathrm{g}$

B

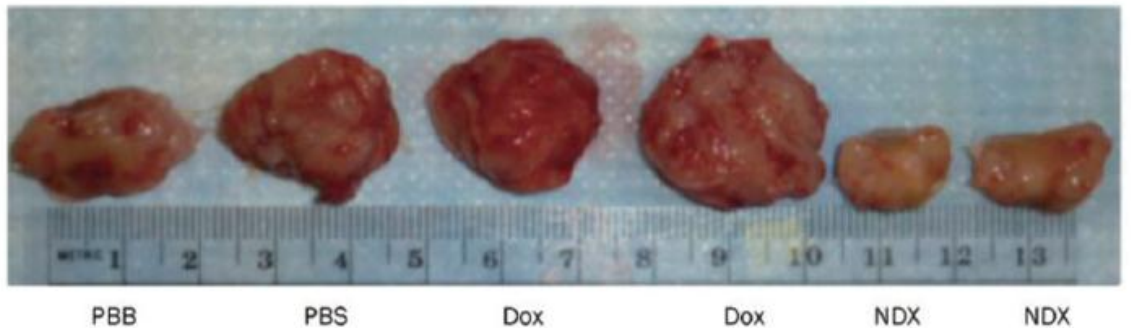

C

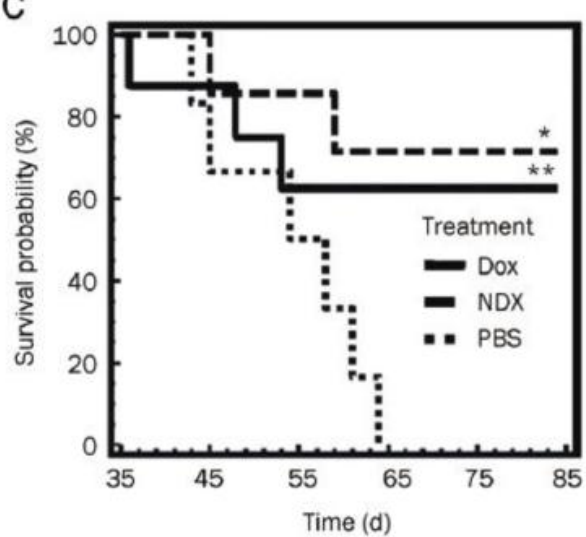

D

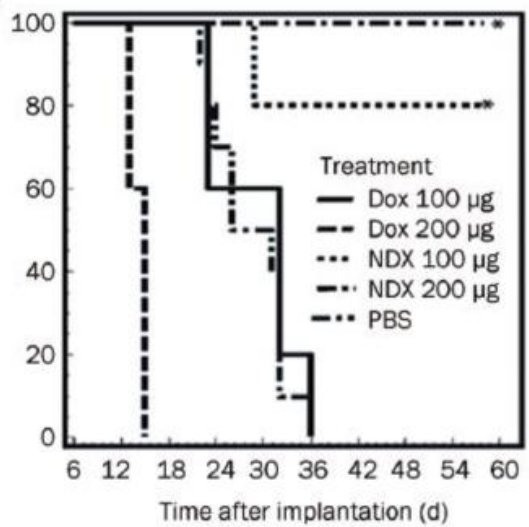

Figure 4. ND delivery of Dox inhibits tumor growth in murine liver tumor models and mammary carcinoma model. (A) Images of livers/tumors from PBS, Dox, ND, or NDX treated LT2-Myc liver tumor bearing mice. (B) Representative images of excised tumors from PBS, Dox, or NDX treated 4TI mammary tumor bearing mice. (C) Kaplan-Meier survival plot for LT2-Myc mice treated with PBS $(n=5)$, Dox $(100 \mathrm{mg})(n=8)$, or NDX (100 mg of Dox equivalent) $(n=7)$ by tail vein injection every 7 d. $* P<0.03$; **P $<0.06$. (D) Kaplan-Meier survival plot for 4TI mice treated with PBS $(n=7)$, Dox $(100 \mathrm{mg})(n=10)$, NDX (I00 mg of Dox equivalent) $(n=10)$, Dox $(200 \mathrm{mg})(n$ $=5)$, or NDX (200 mg of Dox equivalent) $(n=5)$ by tail vein injection every $6 \mathrm{~d}$. $* P<0.003$. [Reprinted from ref. 47 with permission].

In the study of the non-covalent interactions between $\mathrm{ND}$ with insulin, a $\mathrm{pH}$-dependent desorption of the drug from the ND-drug complex was observed. In the experiment, NDs were combined with insulin at a $4: 1$ ratio, and exposure of the ND-insulin complex to alkaline environments led to a significant release of the drug. A 5-day desorption assay in a $\mathrm{NaOH}(\mathrm{pH}$ 10.5) solution and a neutral solution resulted in $45.8 \pm$ $3.8 \%$ and $2.2 \pm 1.2 \%$ desorption, respectively. MTT assays and quantitative RT-PCR (genes Ins1 and Csf3/G-csf, up-regulated by insulin stimulation) indicated that the activity of insulin preserved after desorption, while the adsorbed insulin remained largely inactive. This work developed a therapeutic protein-ND complex with tunable release and pre- served activity [50]. We recently examined the loading of anti-cancer drug 10-hydroxycamptothecin (HCPT) on NDs and its release therefrom as well as the pharmacodynamics of the complex formed. We found that a diluted $\mathrm{NaOH}$ solution $(\mathrm{pH}$ 8.2) can promote the adsorption of HCPT on the NDs and the release of HCPT from the complex. Moreover, ND-HCPT in slightly acidic condition can exhibit a slow release function, and the efficacy on tumor cells is much higher than that of free HCPT [9]. A similar study was conducted by Guan et al. In the experiment, cisplatin, an anticancer drug, was loaded onto NDs by adsorption. In contrast with the above results, it was released from the ND complex in PBS of $\mathrm{pH} 6.0$ at a rate higher than in PBS of $\mathrm{pH}$ 7.4. Accordingly, the authors spec- 
ulate that the ND vehicle would deliver low concentrations of cisplatin in the blood, but release much more drugs after integration into the acidic cytoplasm, thereby reducing toxic side effects. Furthermore, the drug released from the ND complex retains the same cytotoxicity as free cisplatin against human cervical cancer cells [52]. As can be seen, the drug loading and release behaviors of NDs vary because the drugs have different physical and chemical properties. Therefore, to build a drug delivery system, an appropriate $\mathrm{pH}$ condition needs to be selected to effectively control the drug adsorption and desorption on NDs so as to achieve the best treatment effect.

In addition to non-covalent interactions, other studies reported that drug molecules were loaded onto the NDs by covalent binding. For example, in $\mathrm{Li}^{\prime}$ 's experiment, the anti-cancer drug DOX was linked to NDs by the reaction between carboxyl groups of NDs and amine groups of DOX in the presence of EDC and NHS. Furthermore, a cell penetrating peptide TAT was also conjugated to the surface of NDs to avoid premature release and to enhance the intracellular delivery of DOX. Viability determination showed that the ND-TAT-DOX conjugates exhibited a higher cytotoxicity to C6 glioma cells than free DOX [53]. Liu et al. also used the covalent binding method to develop another ND-based anti-cancer drug, ND-paclitaxel. Their results showed that treatment with $0.1-50 \mu \mathrm{g} \mathrm{ml}^{-1} \mathrm{ND}$-paclitaxel for $48 \mathrm{~h}$ significantly reduced the cell viability in the A549 human lung carcinoma cells. ND-paclitaxel was observed to induce both mitotic arrest and apoptosis in A549 cells. However, ND alone did not induce the damage effects on A549 cells. Furthermore, in the in vivo treatment of subcutaneous A549 tumor xenografted SCID mice showed that ND-paclitaxel significantly blocked the tumor growth [54]. In addition, in some researches to build ND-based drug delivery systems, NDs were modified with some chemicals such as $\mathrm{N}, \mathrm{O}-$ carboxymethyl chitosan [55] and benzoquinone [56] so as to facilitate non-covalent interactions between NDs and protein drug molecules. Vaijayanthimala et al. reported that some radiation-damaged NDs could emit strong and stable photoluminescence (red or green) from nitrogen-vacancy defect centers embedded in the crystal lattice [57]. Low cytotoxicity and no sign of photobleaching were found for these NDs, making them well-suited for long-term tracking of a single ND particle in live cells $[4,58,59]$. These functionalized fluorescent NDs may serve as both an imaging system and a drug delivery system for theranostic applications.

Covalent manner and non-covalent manner of building ND-based drug delivery systems have their advantages and disadvantages. A stable drug complex can be obtained in a covalent manner, but its manufacture process is complicated, and moreover it is difficult to remove all the toxic organic solvents introduced during the synthesis process. In addition, the drug complex prepared in this manner usually does not have the slow-release function. In contrast, although the drug bound to NDs in a non-covalent manner does not have high stability, the preparation method is simple and easy, and more importantly, the drug loading can be adjusted by simply controlling some inorganic molecules such as $\mathrm{NaOH}$ and $\mathrm{NaCl}$. The drug complex into the cells does have a slow-release function, reducing the side effects of the drug itself. Therefore, a majority of basic researches at present choose the non-covalent method to build ND-based drug delivery systems.

\section{Perspectives}

The characteristics of NDs, such as optical transparency, chemical inertness, high specific area, robust hardness and excellent bio-compatibility, have provided a series of promising results in basic researches in the field of medicine. However, it should be noted that, like other nanomaterials such as CNTs and graphene [60-65], due to a large surface area and high adsorption capacity, the high adsorption of various components in the environmental media onto NDs may affect their inherent biological effects. It has been found that the NDs might be either nontoxic or toxic to cells, depending on whether serum existed in the culture medium or not. The possible toxicity mechanisms associated with serum proteins and excessive sodium ions delivered into cells by NDs are under further investigation [9]. Therefore, when we build ND-based drug delivery systems, particular attention should be paid to the impact of proteins and ions in the solution on the pharmaceutical efficacy.

Low toxicity and high cellular uptake of NDs in complete cell culture media $[9,16]$ make them an ideal carrier for building drug delivery systems. However, most of the current ND-drug complexes are built as a binary complex system of the NDs and the drug molecule. Unlike CNTs, it has been rarely reported that NDs are simultaneously modified with fluorescent molecules, targeting molecules, and drug molecules to build a multi-functional drug. This is due to the nature of the NDs themselves. Detonation NDs comprise diamond micro-grains with $2-8 \mathrm{~nm}$ in diameter, and the surface area of a single ND is not as large as that of a single CNT. A single ND also does not have high chemical activity or strong non-covalent interaction with other chemicals as a 
single CNT does. However, because of its high surface free energy, NDs rarely exist in a single particle, and usually form clusters of tens to hundreds nanometers in a low free energy, even when they are dispersed in a solution by strong ultrasonication. Since NDs have a very high hardness and nanoscale pores exist among individual particles in the ND clusters, drug molecules or other functional molecules can be assembled on the surface of ND clusters or in these nano-scaled pores in the interior of ND clusters by non-covalent interactions. Various surface or internal distribution of drug molecules or other functional molecules with different sizes can be expected for the ND clusters. Based on the existing studies of the assembly process of several different molecules on ND clusters, a model for the spatial configuration of ND clusters and their assembly process has been proposed [9] (figure 5). According to this scheme, we have succeeded in loading fluorescein- isothiocyanate (FITC), HCPT, and transferrin (TF) onto ND clusters in the order of these molecules' sizes, which is built into a drug (HCPT) delivery system with drug activity, fluorescence function and targeting function. Such a drug delivery system opens up new ideas for the further development of novel ND-based smart drugs which are desirable for their versatility, high efficiency, and low toxicity.
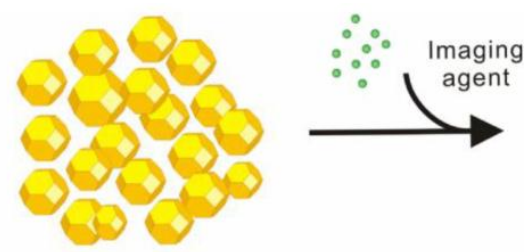

Nanodiamond aggregates
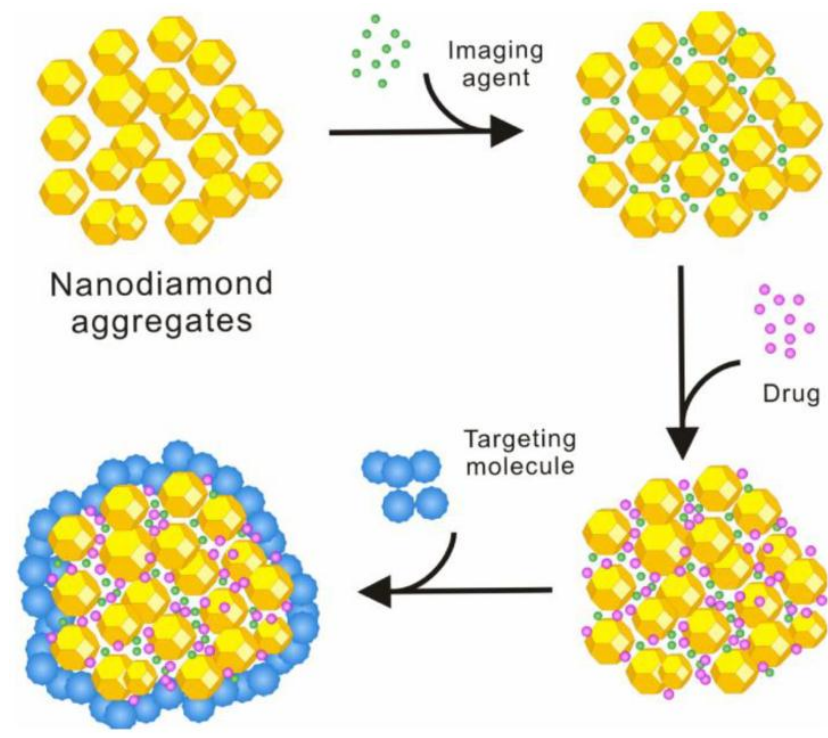

Figure 5. Schematic illustration showing the different loading of different functional molecules on NDs.
Furthermore, the assembly mechanism of various functional molecules such as imaging agents, drugs and targeting molecules on NDs needs to be studied through interdisciplinary researches involving chemistry, physics, materials, biology and medicine. The investigation of the assembly principles and characteristics of different functional molecules on NDs would help to establish the models and theories for building ND-based versatile drug delivery systems, which would serve as the basis for developing a variety of ND-based drug delivery systems with high efficiency and low toxicity to prevent and/or treat various cancers.

\section{Acknowledgments}

This work was supported financially by the Ministry of Science and Technology of China (2012CB825800, 2012CB932600 and 2012CB825805), the National Natural Science Foundation of China (10905086, 10975179, 10905087, 90913014, 61008056, 21105111, 31100716 and 51102272), and the Main Direction Program of Knowledge Innovation of CAS (KJCX2-EW-N03).

\section{Conflict of Interest}

The authors have declared that no conflict of interest exists.

\section{References}

1. Osawa E. Single-nano buckydiamond particles: synthesis strategies, characterization methodologies and emerging applications. In: Ho D, ed. Nanodiamonds-applications in biology and nanoscale medicine. New York: Springer Science+Business Media;2010:1-33.

2. Baidakova M, Vul' A. New prospects and frontiers of nanodiamond clusters. J Phys D: Appl Phys 2007,40:6300-6311.

3. Chang IP, Hwang KC, Chiang CS. Preparation of fluorescent magnetic nanodiamonds and cellular imaging. J Am Chem Soc. 2008;130: 15476-15481.

4. Chang YR, Lee HY, Chen K, et al. Mass production and dynamic imaging of fluorescent nanodiamonds. Nat Nanotechnol. 2008; 3: 284-288.

5. Chao JI, Perevedentseva E, Chung PH, et al. Nanometer-sized diamond particle as a probe for biolabeling. Biophys J. 2007; 93 : 2199-2208.

6. Pramatarova L, Dimitrova R, Spassov EPT, et al. Peculiarities of hydroxyapatite/nanodiamond composites as novel implants. J Phys Conf Ser. 2007; 93: 012049.

7. Huang H, Pierstorff E, Osawa E, et al. Active nanodiamond hydrogels for chemotherapeutic Delivery. Nano Lett. 2007;7: 3305- 3314.

8. Xing $Y$, Dai L. Nanodiamonds for nanomedicine. Nanomedicine 2009; 4: 207-218.

9. Li J, Zhu Y, Li WX, et al. Nanodiamonds as intracellular transporters of chemotherapeutic drug. Biomaterials. 2010; 31: 8410-8418.

10. Schrand AM, Huang HJ, Carlson C, et al. Are diamond nanoparticles cytotoxic? J Phys Chem B. 2007;111:2-7. 
11. Schrand A, Dai L, Schlager J, et al. Differential biocompatibility of carbon nanotubes and nanodiamonds. Diam Relat Mater. 2007;16:2118-2123.

12. Liu K, Cheng C, Chang C, et al. Biocompatible and detectable carboxylated nanodiamond on human cell. Nanotechnology. 2007; 18: 325102.

13. Liu K K, Wang C C, Cheng C L, et al. Endocytic carboxylated nanodiamond for the labeling and tracking of cell division and differentiation in cancer and stem cells. Biomaterials. 2009; 30: 4249-4259.

14. Xing $\mathrm{Y}$, Xiong $\mathrm{W}$, Zhu L, et al. DNA damage in embryonic stem cells caused by nanodiamonds. ACS Nano. 2011; 5: 2376-2384.

15. Magrez A, Kasas S, Salicio V, et al. Cellular toxicity of carbon-based nanomaterials. Nano Lett. 2006, 6: 1121-1125.

16. Zhang XY. Biological effects of three kinds of carbon nanomaterials; Dissertation for the Doctoral Degree. Shanghai: Shanghai Institute of Applied Physics, CAS. 2010: 61-64.

17. Vaijayanthimala V, Tzeng YK, Chang HC, et al. The biocompatibility of fluorescent nanodiamonds and their mechanism of cellular uptake. Nanotechnology, 2009; 20:425103.

18. Yuan $Y$, Wang $X$, Jia G, et al. Pulmonary toxicity and translocation of nanodiamond in mice. Diam Relat Mater. 2010; 19 : 291-299.

19. Zhang XY, Yin JL, Kang Cheng, et al. Biodistribution and toxicity of nanodiamonds in mice after intratracheal instillation. Toxicol Lett. 2010; 198: 237-243.

20. Lam CW, James JT, McCluskey R, et al. Pulmonary toxicity of single-wall carbon nanotubes in mice 7 and 90 days after intratracheal instillation. Toxicol Sci, 2004; 77: 126-134.

21. Warheit BD, Laurence BR, Reed KL, et al. Comparative pulmonary toxicity assessment of single wall carbon nanotubes in rats. Toxicol Sci. 2004; 77:117-125.

22. Muller L, Huaux F, Moreau N, et al. Respiratory toxicity of multi-wall carbon nanotubes. Toxicol Appl Pharmacol. 2005; 207: 221-231.

23. Schrand AM, Hens SAC, Shenderova OA. Nanodiamond particles: properties and perspectives for bioapplications. Crit Rev Solid State. 2009; 34: 18-74.

24. Puzyr AP, Baron AV, Purtov KV, et al. Nanodiamonds with novel properties: a biological study. Diamond Relat Mater. 2007; 16: 2124-2128.

25. Bakowicz K, Mitura S. Biocompatibility of NCD. Journal of Wide Bandgap Materials. 2002; 9: 261-272.

26. Li QN, Xiu Y, Zhang XD, et al. Preparation of ${ }^{99 m} \mathrm{mc}_{\mathrm{C}} \mathrm{C}_{60}(\mathrm{OH}) \mathrm{x}$ and its biodistribution studies. Nucl Med Biol. 2002; 29: 707-710.

27. Li YG, Zhang XD, Li QN,et al. Radioodination of $\mathrm{C}_{60}$ derivative $\mathrm{C}_{60}(\mathrm{OH})_{\mathrm{x}}(\mathrm{O})_{\mathrm{y}} . \mathrm{J}$ Radioanal Nucl Ch. 2001; 250: 363-364.

28. Wang HF, Wang J, Deng XY, et al. Biodistribution of carbon single-walled nanotubes in mice. J Nanosci Nanotechnol. 2004; 4: 1019-1024.

29. Singh R, Pantarotto D, Lacerda L, et al. Tissue biodistribution and blood clearance rates of intravenously administered carbon nanotube radiotracers. PNAS. 2006; 28: 3357-3362.

30. Liu Z, Cai WB, He L, et al. In vivo biodistribution and highly efficient tumor targeting of carbon nanotubes in mice. Nat Nanotechnol. 2007; 2: 47-50.

31. Guo JX, Zhang X, Li QN. Biodistribution of functionalized multi-wall carbon nanotubes in mice. Nucl Med Biol. 2007; 34: 579-583.

32. Yuan Y, Chen YW, Liu JH, et al. Biodistribution and fate of nanodiamonds in vivo. Diamond Relat Mater. 2009;18: 95-100.

33. Rojas S, Gispert JD, Martin R, et al. Biodistribution of amino-functionalized diamond nanoparticles. In vivo studies based on ${ }^{18} \mathrm{~F}$ radionuclide emission. ACS Nano. 2011; 5: 5552-5559.

34. Jia $N Q$, Lian $Q$, Shen $H B$, et al. Intracellular delivery of quantum dots tagged antisense oligodeoxynucleotides by function- alized multiwalled carbon nanotubes. Nano Lett. 2007; 7: 2976-2980.

35. Liu Z, Sun XM, Nakayama-Ratchford N, et al. Supramolecular chemistry on water-soluble carbon nanotubes for Drug Loading and Delivery. ACS Nano. 2007; 1: 50-56.

36. Zhang LM, Lu ZX, Zhao QH, et al. Enhanced chemotherapy efficacy by sequential delivery of siRNA and anticancer drugs using PEI-grafted graphene oxide. Small. 2011; 7: 460-464.

37. Huang $\mathrm{P}, \mathrm{Xu} \mathrm{C}$, Lin $\mathrm{J}$, et al. Folic acid-conjugated graphene oxide loaded with photosensitizers for targeting photodynamic therapy. Theranostics.2011; 1:240-250.

38. Zhang XK, Meng LJ, Lu QH, et al. Targeted delivery and controlled release of doxorubicin to cancer cells using modified single wall carbon nanotubes. Biomaterials. 2009; 30: 6041-6047.

39. Manna SK, Sarkar S, Barr J, et al. Single-walled carbon-nanotube induces oxidative stress and activates nuclear transcription factor- $\mathrm{KB}$ in human keratinocytes. Nano Lett. 2005; 5: 1676-1684.

40. Zhang YB, Ali SF, Dervishi E, et al. Cytotoxicity effects of graphene and single-wall carbon nanotubes in neural phaeochromocytoma-derived PC12 cells. ACS Nano. 2010; 4: 3181-3186.

41. Wang JY, Sun PP, Bao YM, et al. Cytotoxicity of single-walled carbon nanotubes on PC12 cells. Toxicol in vitro. 2011; 25: 242-250.

42. Hartl A, Schmich E, Garrido JA, et al. Protein-modified nanocrystalline diamond thin films for biosensor applications. Nat Mater. 2004; 3: 736-742.

43. Liu Y, Khabashesku VN, Halas NJ. Fluorinated nanodiamond as a wet chemistry precursor for diamond coatings covalently bonded to glass surface. J Am Chem Soc. 2005; 127: 3712-3713.

44. Huang HJ, Pierstorff E, Osawa E, et al. Protein-mediated assembly of nanodiamond hydrogels into a biocompatible and biofunctional multilayer nanofilm. ACS Nano. 2008; 2:203-212.

45. Huang HJ, Dai LM, Wang DH, et al. Large-scale self-assembly of dispersed nanodiamonds. J Mater Chem. 2008; 18: 1347-1352.

46. Lam R, Chen M, Pierstorff E, et al. Nanodiamond-embedded microfilm devices for localized chemotherapeutic elution. ACS Nano. 2008; 2: 2095-2102.

47. Chow EK, Zhang XQ, Chen M, et al. Nanodiamond therapeutic delivery agents mediate enhanced chemoresistant tumor treatment. Sci Transl Med. 2011; 3: 73ra21.

48. Adnan A, Lam R, Chen HN, et al. Atomistic simulation and measurement of $\mathrm{pH}$ dependent cancer therapeutic interactions with nanodiamond carrier. Mol Pharmaceutics. 2011; 8: 368-374.

49. Chen M, Pierstorff ED, Lam R, et al. Nanodiamond-mediated delivery of water-insoluble therapeutics. ACS Nano. 2009; 3: 2016-2022.

50. Shimkunas RA, Robinson E, Lam R, et al. Nanodiamond-insulin complexes as $\mathrm{pH}$-dependent protein delivery vehicles. Biomaterials. 2009; 30: 5720-5728.

51. Ma XW, Zhao YL, Liang XJ. Nanodiamond delivery circumvents tumor resistance to doxorubicin. Acta Pharmacol Sin. 2011; 32: 543-544.

52. Guan B, Zou F, Zhi JF. Nanodiamond as the pH-responsive vehicle for an anticancer drug. Small. 2010; 6: 1514-1519.

53. Li XX, Shao JQ, Qin Y, et al. TAT-conjugated nanodiamond for the enhanced delivery of doxorubicin. J Mater Chem. 2011; 21: 7966-7973.

54. Liu KK, Zheng WW, Wang CC, et al. Covalent linkage of nanodiamond-paclitaxel for drug delivery and cancer therapy. Nanotechnology. 2010; 21: 315106.

55. Wanga HD, Yang QQ, Niu CH. Functionalization of nanodiamond particles with N,O-carboxymethyl chitosan. Diam Relat Mater. 2010; 19: 441-444. 
56. Purtov KV, Petunin AI, Burov AE, et al. Nanodiamonds as carriers for address delivery of biologically active substances. Nanoscale Res Lett. 2010; 5: 631-636.

57. Vaijayanthimala V, Chang HC. Functionalized fluorescent nanodiamonds for biomedical applications. Nanomedicine. 2009; 4: 47-55.

58. Yu SJ, Kang MW, Chang HC, et al. Bright fluorescent nanodiamonds: no photobleaching and low cytotoxicity. J Am Chem Soc. 2005; 127: 17604-17605.

59. Fu CC, Lee HY, Chen K, et al. Characterization and application of single fluorescent nanodiamonds as cellular biomarkers. Proc Natl Acad Sci. 2007; 104: 727-732.

60. Hurt RH, Monthioux M, Kane A. Toxicology of carbon nanomaterials: status, trends, and perspectives on the special issue. Carbon. 2006; 44: 1028-1033.

61. Zhu Y, Ran TC, Li YG, et al. Dependence of the cytotoxicity of multi-walled carbon nanotubes on the culture medium. Nanotechnology. 2006; 17: 4668-4674.

62. Casey A, Davoren M, Herzog E, et al. Probing the interaction of single walled carbon nanotubes within cell culture medium as a precursor to toxicity testing. Carbon. 2007; 45: 34- 40.

63. Casey A, Herzog E, Davoren M, et al. Spectroscopic analysis confirms the interactions between single walled carbon nanotubes and various dyes commonly used to assess cytotoxicity. Carbon. 2007; 45: 1425-1432.

64. Zhu Y, Li WX, Li QN, et al. Effects of serum proteins on intracellular uptake and cytotoxicity of carbon nanoparticles. Carbon. 2009; 47: 1351-1358.

65. Hu W B, Peng C, Lv M, et al. Protein corona-mediated mitigation of cytotoxicity of grapheme oxide. ACS Nano. 2011; 5: 3693-3700. 